\title{
La distocia de encajamiento de causa secundaria en la presentación de vértice
}

\author{
Por el doctor JOSE MIGUEL VARGAS PAEZ
}

\section{ALGUNAS HISTORIAS CLINICĀS}

Entre tas muchas historias clinicas que sirvieron para demostración y esti. -mulo de este trabajo, sólo publicamos. para no ser prolijos, las siguientes: Nos. 1.757, 1.830, 2.470, 1.727 y -554.

La distocia de encajamiento reconoce, como es naturali diferentes causas que por razón de su origen las divi. diremos en causas maternas fetales $y$ ovulares.

Ahora bien, la distocia según su etio logia, puede obedecer a causas primitivas, distócicas en sí y desde-un principio y a causas secundarias ya no absolutas, sino relativas, que pue den poner a prüeba la habilidad $y$ criterio científico del obstetra, cuando eñ el desempeño de su profésión tro pieza con uno de esios accidentes tan desagradables.

Son distocias primitivas, aquéllas que no permiten la realización del parto por las vias genitales, como la distocia originada por una pelviviación: estrechez o deformación por acción directa del bacinete óseo y que entra en el dominio o capítulo de las distocias óseas; o bien, cuando el bacinele óseo normal se encuentra vi ciado o comprometido relativamente. ya sea por Iratarse de una presenta. ción viciosa, o porque la cabeza de dimenisiones mayores a to normal, no puede electuar su paso por el área del estrocho superiar: o porque aủn con bacinete y cabeza de dimensiones normales, existe vicio de encajamiento por tumelacción peri-fetal, peri-uterina, para uterina $y$ endo uterina, como er el caso de fibiomas, quistes o placenta, previos.

Pero en cambio, existe otro grupo de causas que impiden o dificultan el encajamiento-de la presentación, no obstante de tratarse de casos en los cuales la petvis $\in s$ normal, las dimensiones de la cábeza normales y no existir ninguna de las causas anotadas en el grupo de las distocias que denominarros primitivas. A este grupo dé distocias que hemos denominado secundarias y que ha originado nuestro estudio, es a las que queremos referirnos en particular; ya que la distocia primitiva es única, no ádmite discusión y la conducta obstétrica y su terapeutica están definidas. Por el contrario, la distocia secundaria es de interpretación diferente, según el criterio de cada individuo exige por lo tanto,-un mayor conocimiento de causa y efecto, se presta con mayor factlidad a la discusión, por consiguiente exige más cuidado y mayor criterlo obstétrico, cuándo por rázón de constituir una distocía y tener que resol. verla, hay que adoptar una conducta racional $y$ eficaz. 
El grupo de distocias que nos ocupa $y$ al que hemos dado el apelativo de secundarias, constituye un buen núme ro y la palabra secundaria con que lo hemós designado no le resta ímpor. tancia, ya que son por lo demás, bas. tante frecuentes y muchas veces cons thuyen unc verdadero probleme obstétrico dr dificil solución. Las dividi. mos en razon do su origen, en distocias maternas, fetales y ovuiares y procu raremos hacer de ellas un grupo definida y claro. Las principales son:

ja En la madre, la distocia útérina fisiopátológica, en el cuerpo, segmen. to o cuello; dinámicas en las discine: cias o inercias uterinas; y mecánicas en las anteposiciones irreductibles y tumores del cuerpo.

$2^{a}$ Fetales. Los trastomos del lisio logismo de la cabeza, en los cuales se presenta mala orientación y acoma dación de ésta, con deflexión incom. pleta del polo celálico, sin llegar a - ser presentación de frente o cara y en las variedades de posición como en las occipito-posteriores.

$3^{i}$ De los anexos. La iuptura prema tura e intempestiva de lá bolsa, la bre vedad del cordón umbilical y el hidramnios.

Por ultimo, existen causas secundarias mixtas, cuando se asocian dos $c$ más de las mencionadas, y cợusas iñclasificables, cuando no obstante no apreciarse causa visible y precisa, el - encajamiento-no se electúa, sin que podamos ver claramenté dónde estri. ba-la dilicultad.

\section{Descripción y explicación}

a) Al estudiar el mecanismo del parto, hay que tener en cuenta tres ele. mentos-fundamentaies: ol - motor del parto, el objeto del parto y el canal dèl parto. De: la sinergia funcional de estos tres elementos duranle el trábajo de parto, so consique el parto fisio Jógico; normal o eutócico. Pero si uno - varios de dichos elementos se desvian de la linea funcional fisiologica, aparece el parto patológico, dificil o distócico.

Siendo el útero y ta buena constitución de la pared abdominal, la causa -que impele al feto, su salida del-vientre materno, la disfunción de êstos motores se nos presenta como la primera causa de la distocia de encajamiento:

En el útero, la distocía bien puede ser anatómica o tisiológica. El útero - puede adoptar por diferentes causas de todos conocidas, posiciones desventajosas, como anteversiones exagera. das, desviaciones taterales, etc., que llevan al cuello hacia distintas posiciones anormales, que impiden la coinci. dencia del ejé uterino con el eje del conducto pelvigenital, - y -por constguiènte, desvian el ejo que la presen. tación debe-seguir, impidiendo su encajamiento circunstancias que en ve ces se pueden corregir fácllmente, pero que otras obligan a electuar una inter. vención obstétrica; esta clase de disto cias es puramente mecánica o anató mica.

Pero la fibra muscular uterina, puede ser asiento de disturbios funcionales que afecten la motilidad de todo el órgano o de una de sus-partes, apareciendo, como es natural, la distocia dínámica, que unas veces se puede vencer médicamenté. pero que en oca. - sionos se hace de muy dilicil solución. Dentro de este tipo de distociás, en contramos las inercias, primltivas o secundarias, cuando hay defecto do contracćlón por debajo de la normal; 
- his aiscmecias en las que se presentan contracciones excesivas o desordenadas, es decir, distocias en las que se encuentra un defecto de la contraeción de tipo, por encima de la normal - hiperdinamia uterina, que puede alectar la contracción en sus tres formas: frecuencia, intensidad y tono basal produciendo los tres sindromes: taquisistolu, hipersistolia o hipertonía, la que bien puede localizarsé en todo el órgeno o alectar uno de sus segmentos cuerpo, segmento inferior o cuello. Todas ellas constituyen disto cia, especialmente la última, és decir. la hipertonia, pues en ella se halla muy elevado el tono basal de la musculatura uterina, dando como resultatado contracciones que aunque poten. tes, y dolorosas en extremo, no son efoctivas para el progreso del parto. toda vez que éste se halla intimamente ligado a la amplitud de la_sístole uterina o lo que es lo mismo, a la amplitud de la diferencia entre el tono bosal $y$ el acmé de la contracción; cuando esta diferencia es pequeña, el perto no progresa y nos encontramos ante un estado en el cual la presentación no avanza; es una distocia que si se instala antes del encajaraiento. constituye distocia de encajamiento secundaria a un estado de hipertonía uterina. En esta clase de distocias, -podemos encontrat desde un ligero aumento del tono basal, hasta el cuadro dé la contractura o tétámos uterino. pasando por varios estadós intermedios, tales como las contracciones- espasmódicas, los espasmos parciales en el cuello, anillo de Bandl, erigen de los ligamentos redondos, eñ-los cuernos ulerinos o en tódo el cuerpo del útero, inclusive el anillo de Bandl, has ta llegar al espasmo total-icuerpo, seg. mento y cuetlo), lo que constituye el tétanos o contractura del útero, casos todos en los cúales el parto se detiene si no se logra vencer la dificultad, poniendo en grave peligrc tanto a la madre como al fëto. Por último, cuando la propiedad retráctil del músculo uterino se altera hacia el acortamiento permanente, se presenta la distocia de retracción a la cual so puedo llegar después de un periodo de contraccio. nes espasmódicas, de espasmos totales o parciales o con la aplicación des. medida de ocitócicos.

b) El cuello del útero suele ser con frecuencia, asiento de distocias secundarias que impiden el encajamiento, y ellas, como las del cuerpo, pueden ser anatómicas y funcionales. Parece existir un antagonismo funcional entre el cuerpo y el cuello por influjo de su inervación, mediante el cual, a la contracción enérgica del cuerpo, corresponde una -actitud pasiva del cuello, que se deja dilatar paulatinaménte; pero el-antagonismo puede invertirse de tal mānera que el cuello se contraiga espasmódicamente, produciendo sobre el cuerpo un influjo paralizador, o bien dándole un carácter espasmódico: es lo que se denomina disociación de la sinergia funcional; poro lambién la distocia puede quedar localizada exclusivamente en el cuello, presentándose como única manifestación el espasmo total o de uno de los orificios, interno o externo.

Adernás de las disfunciones moto. rás, en el cuello pueden presentarse alteraciones congénitas o procesos anatómicos que impidan su dilatación. como el tabicamiento, la hipoplasia. desviaciones, obliteración, edemas, hipèrtrofia, aglutinación y rigidez, todo to cual puede conducir a la imposibi- 
liajad o a la dificultad del encajamiento. Es decir, lo físiólógico es que la dilatación del cuello - sea efecto de la contracción uterina, y que la causá del encajamiento también sea la contracción pero sea porque se destruya esi sinergid o sea porque haya dis. is ir en el cuello que le impide res. - ador a la acción de la contraccion. 1. ciarto es que en la práctica con mictio frecuencia en estus casos en que hay alteración en el canal cervi. cal è encajamiento se compromete y la prueba está en qué en los indicés estadísticos no es raro hallar scesárea por distocia del cuello, sin otra çusa especial.

c) Por parte del feto, se pueden presentar causas de distocia de encajamiento, sin tener en cuentr las presen. taciones -viciosas como las de cara y frente. Ya hemos dicho que la presen. tacion con el objeto de hacer coinci: dir sus diámetros con los del estrecho superior para efectuar el encajamiento debe realizár movimientos apropiados de flexión, rotación y acomodación y movimientos de báscula que le permitan deslizarise dentro de la pelvis. como son los movimientos de asincli. tismo anterior o posterior. Pero pue. de suceder que estos movimientos pre faratorios no se efectúen o lo hagaan en forma exagerada, dando-lugar a la distocia dé encajámiento de corusa secundaria.

A! sobrevenir la contracción utent. na en el momento del parto, aquélla sorprende a la cabeza por encima del estrecho superior en actitud indiferento: entonces bajo su influjo se produce el movimiento de flexión. Luégo, la car beza rota haciendo colincidir la sutura sagital con uno de los diámetros, trans. verso u oblicuo y acomodando sus cir- cunferencias menores a las circunierencias mayores de la pelvis. Cuando el fisiologismo de estos movimientos se altera, se presenta mala orientación y acomodación, lo mismo que defle. xión incompleta. intermedia entre sincipucio-y trente. Lo mismo -sucedo cuande la rotacion en vez de llevar el u cirital hacia adelante. lo lleva haeir atrás a variedades de posición posteriores; en las cuales ta cabeza nuncá está bien flejada, ofreciendo por consiguiente diámetros mayores que dificultan el encaiamtento y hasta pueden presentarse casos en que se coloća en posición anteroposterior pór éncima del estrécho, cuando lá pelvis es de forma pitecoide o androide.

De la misma manera, pueden presentarse a distocia los movimientos exagerados de asinclitismo en las pre sentaciones que algunos autores llaman de parietal anterior, o posterior, pues en estos casos la cabeza desviada lateralmente, choca contra una de las salientes del estrecho que bien puede ser el promontorio cuando el asinclitismó es posterior o el pubis. cúando lo es anterior, produciéndose en esta forma una imposibllidad para el encajamiento.

d) En los anexos, la causa de distocia puede estar en la bolsa, en el-cordón o en el líquido amnlótico. Nadía desconoce el papel dilatador que ejerce la bolsa de las aguas sobre el cue11o, por exeltación o por piosibn, al in sinuarse en su canal. Cuando la ruptura de la bolsa se presenta prematuramente, sobre todo en las primigestantez añosas, se producen disturblos en lá dilatación, desde la dificultad de hacerlo, hasta el espasmo cervical y muchas veces vemos cómo ol úte ro, por ruptura de la bolsa o por ad. 
herencia intima del polo inferior del huevo al orificio cervical, entra en contracciones espasmódicas, discinecias 0 inercias, sudiendo llegar hasta el tétanos úterino y entonces crear una distocia de encajamiento de causa secundaria, desencadenada por la rup tura prematura de la bolsa.

e) Factor do mache imporancia pa ra el buen meransmo del parto, lo cncontramos a cordon umbilical ya que de él depende en gran parto la vida del feto. Sabemos que la presen. tación para encajarse y descender, ne. cesita un radio de acción que le permita progresar, y este radio de acción se lo dá el cordón. Pero cuando por alguna circunstancia dicho cordón re. sulta demasiado corto, se constituye en un freno que le impide al teto avan. zar por el canal pelvigenital, constituyéndose en esta forma, en una distocia de encajamientc $y$ en un grave peligro para la vida del feto, y esto se comprueba en casos de cortcrad absoluta o relativa.

f) Por último, el líquido amniótico puede constituir causa de distocia cuarido en razón de su gran cantidad (hidramnios), es causa perturbadora del fisiologismo de la cabeza, prod:1 ciéndole movilidad exagerada, o bien por la gran distensión que ex:ge:a la fibra uterina, la pone en condiciones inadecuadas para cumplir sus funcio nes contráctil y elástica, dando como resultado en ambos casos una disto cia de encajamiento de corusa secun. daria.

Todas las causas anotadas pueden obrar individualmente o bien asociarse para producir distocia dé cousa mixta. Pues, además de ellas, hory causas que so nos escapan al conoci miento, pero que pueden ponerse de manifiesto en casos en que ei encajamiento no se produce, sin que podamos determinar la razón de ser de la distocia.

Las dificultades para el encajamiento que hemos enumerado en los párofos antericres, en mujeres con pelvis de dimensiones normales y con letos de tanraño nomal en presentr. cion de ventice, -constituye to que nos. otros hemos denominado distocia de encajamiento de causa secundaria en la presentación de vértice.

- En las historias clínicas que presentamos a continuación, cuya veracidad èstá comprobada, porque los origina: les existen en archivos muy serios, presentamos la base de nuestro estudio y la comprobación de la existenciá de la distocia de que nos ocupamos.

\section{HISTORIAS CLINICAS \\ Y COMENTARIOS A CADA UNA DE ELLAS}

\section{Historia número 1.830}

Protesor Mojica $M$.

1951. 32 años. Multigestante de ocho meses.

Cesárea por rigidez del cuello.

Antecedentes obstétricos: Dos embarazos terminadós ambos por ceśárea, por falta de encajamiento y rigidez del cuello.

Examen obstétrico: Abdomen normal; útero; 28 ctms., ovoide, blando, regular, longitudinal; tonicidod normal; liquido amniótco, normal; tê̄o úntco, vivo; vértice, Oll insinuada; palpación de Pinard, favorable. Pelvimetría notmal.

Inició trabajo el 24 , a las 20 horas. 
Tacto vaginal: El 25 a lás 10 y 25 : Julva y vagina, normales; cuello, inompletamente borrado, cerrado, esasmódico. Se ordena atrinal y dóricor 1 las 21 horas del 25, tacto vaginal: lulva y vagina, normales; cuello cenral, grueso, permite el paso de un de lo; bolsa integra. Plana. Presentacion nsinuada en OIDA. Ruidos cardiacos etales, normales. A las 23 horas del 5. la paciente sigue en las mismas ondiciones, no obslante la aplicación le atrinal y dórico, repetidas ocasio es; el cuello está grueso, borrado, con in ctm. de dilatación: la presentación le vértice, apénas fija. Las contraccio es cada tres minutos, intensas; los uidos fétales, "normales. En vista de a-uniformidad del cuadro durante mas le 24 horas y teniendo en cuenta el mtecedente de dos cesáreas, se opta or cesárea por tercera vez. Se practia cesćréa segmentária transperitoieal con sección de las fibras del segnento. Se utiliza el fórceps de Simpon para la éxtracción de la cabeza. it feto respira espontáneamente.

Alumbramiento, normal; hemorragia iterina de consideración. Sutura del tero y cierre de la laparotomia en la orma acostumbrada. Se obtiene un sto prematuro de 2.100 gramos, en uenas condiciones.

Salida del hospital, seis dias más arde, en perfectas condiciones.

En este caso, como se desprende de a Kistorla, la cesćrea fú practicada or distocia de encajamiento de causa -cundaria, debida a rigidez del cue: o, lo cual habia motivado las cescieas anteriores $y$ que no lue vencida or el trabajo prolongado y la aplicaión de espasmoliticos, la intervención - elección tenía que ser la cesárea, a que la falta de dilatación del cuello impedici la aplicación de un aperato de torceps, a lo cual so sumabri el antecedente de las operaciones anterio. res. En está intervención. como se ve, no hubo morbilidad ni mortalidad ma. terna ni letal.

\section{Hintoria número 554}

Prolesor Moiica.

1951. Multigestanie a témino.

Fórceps alto por trabajo prölongado y sutrimiento tetal.

Antecedentes obstetricos: Tres partos de siete, ocho y nueve meses, res pectivamente.

Examen obstétrico: Utero, normal, de 32 ctms. Abdomen, normai; embarazo a término, normal; leto único, vivo; vértice. OI, insinuado; palpación de Pinard, favorable. Pelvimetria, 24, 28, 30, 20.

Iniciación del trabajo, el 28 a lás 11. Tacto vaginal, a las 14 horas: Pertreo con desgario antiguo. Vulva y vagina, normales. Cuello -grueso, dilaracion, nueve ctms. Membranas integras. Pro sentacion tija. A las 14, ruptura espontánea de las membranas con salida de méconio. Los ruidos letales se hacen lentos. Se aplica coramina, y los ruidos continuan regulares. El 28, a las 17, tacto vaginal: Cuello blando. dilotación completa. Ruidos acelerados. A tas 18 y 30: Las mismas condiclones, expulsión de meconio y ruidos malos, por lo cual se résuelve aplica$X$ ción de forceps alto. Provia aplicación de coramina y mejoramiento de los ruidos, se hace caplicación de fórceps para OIA. La extracición del teto tue fácll. La torna resultó oblieua. Se obthene un leto que sespirc después de aplicarle conaléplicos $\mathbf{Y}$ vitamina $\mathbf{K}$. 
Longitud del cordon, 15 ctms. Puerperio, normal. Salen del servicio en buenas condiciones.

Comentario. Multigestante con partós normales en la cual ta pelvis es normal y no se encuentra distocia ma temo-fetal. Existia distocia de encajamiento de causa secundaria. por biovedad absoluta del cordon. Por sufri. miento fetal y filia de encajamiento se procedio a la eplicación de forceps qlta, solucionándose la diticultad è forma satistactoria con la obtención de un foto en buenas condiciones y sin producción de lesiones maternas: $=$ ni morbilidad fetăl.

\section{Historia número 2.470}

\section{- Profesor Moíica M.}

$x$

1951. Multigestante a término.

Cesárea por suffimiento fetal y tra bajo prolongado

Antecedentes obstétricos: Tres-partos normales.

Examen obstétrico: Abdomen, nor: mat útero, 32 ctms., normat; embarazo a- término normal. Feto único, vivo; vértice, $O D$, insiñuada. Palpación de Pinard tavorable. Pelvimetria: 23, 26. $31,21$.

Iniciación del trabajo el 22 a las 12 . horas.

Tació vaginal a las $20=$ y 45 , del-23: Genifales, normales; cuello central, blando, dilatación total, membranas rotas -Ruidos lentos, expulstón de me conto: Presentación insinugda en ODT. A tas 22 horas, presentación $O D A$, sin encajar; huesos con gran cabalga. miento $y$ bolsa-serosanguinea muymarcada. Expulsión de meconto: Rutdos, buenos. Por el sufrmiento tetal y falta de eneajamiento, se decide hacér cesárea-segmentaría por el méto- do de rutma. Operación sin inciden tes. Se obtiene un feto que nace en apnea, pero respira poco después, se nota deformación de la zona parietal izquierda signos de asinclitismo exagerado que ocasionó la falta de encajamiento por mala aromodacion do la cabeza. Salen en buena: contiriones.

Comentario. En esto promte con partos nomales a termino, !a falta de encajamiento se dobe a un asinclitismo exagerado, pero esta no es una verdadera distocia irreductible y conside ramos que en estas condiciones es fátible la realización del parto-por vía baja mediante aplicación de fórceps que hubiera podido intentarse y en caso de tracaso, haber recurrido a la cesárea pues lo más corriente es que el forceps vence la distocia máxime tratándose de multigestante -con pelvis normal.

\section{Historia número 1.757}

Profesor Mojica M.

Multigestante a térmiño.

Fórcops por sufrimienta fetal' y tra. Fio prolongado:

Antecedentes obstétricos: Cuatro partos a término y un caborto de dos y medio meses.

Examen obstétrico: Abdomen, normal; utero, 31 ctms; hipertonia uteriná: feto único, vivo; vértice, OII, insinuado. Palpación de Firard lavorabie. Pelvimetría: $23,26,29,19$.

Iniciaçión del trabajo: El 17 a las 20 horas. La prciente llega al sorvicio con hipertonia uterina $\theta$-inercia se: cundaria. En la casa le fueron aplicadas tres ampolletas de pitocín. Ruidos tetales lentos. $\mathrm{Se}$ lo aptica coramina. atrinal, dórico. 
Tacto vaginal a ias $12-y 30$, del 18 : Vulva y vagina, normales: cuello con dilatacion completa; bolsa plana que se rompe artificialmente; presentación insinuada en OIT. Como los ruidos continuan lentós $y$ se hacen intermi. temtes. se intenta hacet una anlicacion d. torcus poto como en in interven la cabeza se sute for encina (i) estecto superior, st suspendo $y$ su aplica nuovamente coramma. Tac to vaginal a las 15 y 30 : Paredes vaginales congestionadas. Cuello con dila. tación sompleta $\bar{i}_{--}$excepto un reborde pequeño, que se nota al lado derecho. Presentación en OIT encajada, un poco por debajo del estrecho superior. No se toca promontorio. Bolsa rota. Los ruidos fetales han meiorado $\mathrm{cc}$ las aplicaciones de analépticos realizadas. A las 15 y 45 , aplicación de fórceps por sufrimiento fetal $\mathrm{y}$ trabajo prolongado. Presentación en OIIT. Tee nica del doctor Ramírez Merchán, in. troduciendo primero la fama derecha invertida por el lado izquierdo, se ha. ce girar por debaja del pubis hasta llevarla a la extremidad derecha del diámetro fransverso. La presentación ha rotado a O. P. y se ha desplazado hacio arriba, quedando por encima del estrecho superior. Se introduce la segunda rama por la extremidad iz. quierda del diámetro transverso. Con facilidad se consigue el descenso de la presentación. Se obtiene un feto que respira espontáneamente. Toma ideal. Madre e hijo salen en buenas condiciones del hospital, a los pocos días.

Comentario: ¿Por qué no se produjo el parto espontánea en esta paciente. siendo una multigestante con pelvis normal y el contenido uterino no era despropotcionado a la pelvis materna? Existe la presencia de una hipertonia uterina con mercta paradogica p:oducida por el uso extemporáneo de ocitócicos que al excitar la fibra uterina $y$ activar las contracciones, llevaron a la matriz a la hipertonia, elevaron el tono basal de la fibra muscular $y$ perturbaron "la dinámica uterina, haciendo que su trabaio fuera exagerado e inutil. En estas condiciones vemos cómo ante un trabajo que se pro. longa y frente al sufrimiento fetal, la -aplicación de un fórceps por encima del estrecho salva las circunstancias, produciéndose el parto por via baja y con resultado muy satisfactorio.

\section{Historia número 1.830}

Prólesor Acosta.

y 1951. Primigestante a termino. Pa ciente de 33 años.

(c)

- Cesárea por sufrimiento fetal y edad de la paciente.

Examen obstétrico: Abdomen, normal; útero, 31 -ctms., normal; liquido: normal. Feto único, vivo, vértice $\mathrm{OI}$, insinuada. Palpación de Pinard: favorable: Pelvimetria: $23,26,31,21$. Em barazo, normal.

Al ingresa la paciente relata que to fueton suministradas, antenoche. dos cápsulas de quínina y anoche otra.

La iniciación del trabajo no se procisa.

Tacto vaginal, el 29 a las 12 y. 15: Perinea, vulva y vagina normales. Cua Ito central, borrado; blando, dilatación de cinco ctms. Membranas rotas. Pro sentación móvil en OIA. Ruidos fetaLes, normales. A las 16 y 30 , tacto va ginal: Cuello borrado, dilataclón de siete ctms.; bolsa rola, vértice insinuado OlA. Ruidos tetales, buenos. A las 22 y 40: Cuello ligeramente grueso, 
borrado, dilatación de seis ctms., bolsa rota. Presentación OIA, rechazable; ruidos fetales, buenos. Paciente que está en trabajo deside el 28 , con dolores muy irregulares, que le han administrado tres cápsulas de quinina, en dosis fraccionadas. Primigestante de 33 años con émbarazo a término. En vista del trabajo y de los exáme nes repetidos $\sin$ que se note tendencia al encajamiento, se pone on conocimiento del Jefe de Clínica er turno, quien encuentra sufrimiento fetal, por 10 cual decide terminar el parto por cesárea. Anestesia raquídea. Cesárea segmentaria transpe ritoneal por el método de rutina. Se extrae un feto que respira espontáneamente. Hay abundante meconio en la cavidad uterina. La madre y el niño salen de la sala en buenas condiciones. Postoperatorio, normal. Abandonan el servicio pocos días después.

Comentario. En esta paciente et encajamiento no se efectuó, no por una distocia mäterno fetal, puesto que no se aprecia desproporción y la pelvis materna es normal. Existia, pues, otra causa de distocia y por consiguiente se trataba de una distocia de encajamiento de causa secundaria, ocasio nada por la ruptura prematura de la bolsa que produjo un trabajo irregular y una falta de dilatación del cue Ho. A ello, hay que agregar, la circunstancia de tratarse de una primigestante añosa en las cuales el parto espontáneo es mucho más dificil por irregularidad del trabajo y la poca adomptabilidad de los tejidos para la función que -deben desempeñas en el partó. Pero no_obsianto estas circuns tancias, el parto hubiera podicio inten tarse por vía baja, recurriendo a la cesárea en caso de fracaso.
Evalución. pronóstico y conducta en la distocia de encajamiento de crusa secundaria en la presentáción de vértice. Resultados.

En la mayoria de los casos el médico no puede prever la posibilidad de la presencia de una distocia de este tipo antes de iniciado el trabaio do parto, y aún mas, antes de que haya transcurrido algún tiempo de iniciado. Partimos de la base de que en este tipo de distocia se encuentran objetivamenie condiciones favorables para la realización del parto en forma espontónea.

La terminación del embarazo por el parto normal, constituye la máxima aspiración de la fisiología obstétrica. pero no podemos considerar el parto como un acto puramente mecánicc. puesto que es una función orgánica, y como tal, está regido por leyes especiales, todo lo cual constituye la fisiolcgia obstétrica. De modo que este es un acto esencialmente fisiológico, desti. nadó a dar cumplimiento a leyes birlógicas y naturales, al igual que todas las funciones orgániças constituyen actos fisiológicos.

Pero sucede a menudo que la tisic logía se ve alterada en todas o en una de sus partes, desviándose en -su función y constituyéndose en un acto anormal o patológico que en nuestro caso lo constituye la distocia.

En la distocia secundaria, el traba. to se inicia por lo general en forma as parecer norinal, pero no tarda en presentársenos como un hecho patológt. $c o$, que se traduce en la falta de enca. jamiento de la presentación y que pue. de evolucionar on dos formas: o bien et parto se prolonga excesivamente con un trabajo regular o exagerado 
que conduce a la mercia secunadica por agotamiento físico de la tibra muscular uterina, paralizándose de esta manera el trabajo, o bien ante el obstáculo surgido; el cuadro clínico cambia, translormándose ese fraboyo regular en un trabajo intenso, desespe. rante, tanto para la madre como para el obstetra. En estas condiciones si el encciamiento no se produce, a pesur del esfuerzo de la contracción-uterina. puede coriducir especialmente - si la bolsa se ha róto, a un accidente tan grave como es la inminencia de ruptura del útero, cuyo désenlace puede ser la ruptura declarada, o a la para. lización del trabajo por una inercia paradógica, con contractura y tétanos uterino y todo esto con una con: secuencia fatal: el peligro de la vida de la madre y-la muerte del feto, casi inevitable.

En otras ocasiones más favorables y gracias al estuerzo muscular del útero. que redobla su potencia ante el obs táculo, máxime si se trata de pacientes con bacinete normal y teto de ta. maño y presentación normales. el par: to puede realizarse espontáneamente, y a expensas de un gran sufrimieno materno y fetal que puede producir riesgos en éste o su muerte in utero-o en un futuro próximo o tardio par iraumatismo obstétrico inherente al parto. Mas no siempre la distocia pusde ser voncida en esta forma y el mé dico tiene la obligación sagrada de resquardar las dos existencias que se le han puesto en sus manos, como a un apóstol del blen, a-más de sei ūn funcionario social, especialmente cuan do es obstetra, que vela dia y noche por la madre y por el hljo, bases de * la sociedad y de-la nacionalidad.

En circunstamctas tales, cuando en - f curso de un trabajo de parto, surge una complicacion de las que venumics estudiando, lógicamente el pronóstico maternofetal se hace desfavorable, ya que en condiciones extremas se puede llegai a la muerte de uno de los dos seres, o lo que es peor, a la de ámbos. si es que el parto no se realiza des. pués de un trábajo prolongado, con éxito para uno y para el otro. Y aqui es donde se pone a prueba el criterio, la zagacidad y la pericia del obstetra. que abocado a un problema de esta naturaleza, debe adoptar una conducta franca y responsable: Aqui es donde entran en fuego la responsabilidad y-la conciencia, en primer lugar para - ciceptar y comprobar el prońśstico desfavorable, y en segundo lugar, para adoptar la conducta obstétrica que $I$ su modo de ver sea más lógica, rczonable e inocua.

Como primera medida el obstetra ha de tener en cuenta las consecuencias que se derivan de una conducta me ramente expectante o de su inversa, el intervencionismo desmedtdo. Ya dijimos qué la expectación irresponeáble - el abandorio, pueden conducir a la ruptura uterina con fállecimiento $\mathrm{ma}$. terno y fetal, a la paralización del trobajo en una $u$ otra forma con perdida de la vida del fetó y grave peligro de infección de la madre, que una vez is tas las membranas esté expuesta a la invasión de gérmenes a la cávidad del útero y de allí a todo el organismo, o a la muerte del teto exclusivamente con pérdida para lá soctedad. Pero ea el caso meños grave de que parto lograrar, a pesar de la distocia, realizarse espontáneamente después de um trabajo prolongado y excesivo, puede suceder que el feto muera en tै. o qu.s sl nace vivo. por el suírimiento tranco a que se vió sometido, nazca con lesto- 
nes graves que le quiten la vida re cién nacido, o le dejen en condiciones de inferioridad en relación con sus $\mathbf{s} €$. mejantes.

¿Qué conducta debe entonces seguirse cuando durante el parto aparece is: distocia? Naturalmente la conduct: deponde en general de la causa, cuan do ésta se puede precisar, y asi tern mos dos grandes grupos de condu tas: médicas y obsténıcas. Cuando : criterio del médico considera que distocia puede yugularse médicame.! te, debe intentar todos los tratamien. tos que estén a su alcance, con el fin de évitar cualquier intervención qú rúrgica. Y así, cuando se trata de dis. tocias por defecto de la contracción $\mathrm{d}$ útero, en los casos de inercia primit. va o-secundaria, puede y debe em plear los médicamentos excitadores $d$ la contracción uterina, es decir los oci tócicos. Pero el ocitócico de elección no ha de ser cualquiera de los fárme: cos a los que se les reconoce acción sobre la contracción uterina, sino at mejor $y-e l$ más indicado para este pe. ríodo del parto, como son los extractos del lóbulo posterior de la hipófisis, ta les como el pitocín y sus similares. Estos extractos obran produciendo un estímulo de la fibra muscular lisa, ez pecialmente del miometrio, de tal suer: te que en un útero en inercia primitiv o secundaría o con contracciones muy débiles, obran produciendo reactiv ción y regularización dé las contracciones, ya que estos extractos son los estimulanfes naturales $-y$ fisiológicos del útero. Claro está quẹ -cuando la distocia obedece a contracciones alte radas por exceso, es decir, cuando se trata de polisistolia o hipersistolia ut rinas, entonces los-ocitócicos están formalmente contraindicodos, loda $v=z$ que no-harión más que cumentarla.
Fero si tratacia la inercia con ios es timulantes de la contraccion uterina. ésta no obedece al tratamiento racio. nal que se ha hecho, entonces habrá necesidad de emplear uno de los métodes quirúrgicos que el obstetra elegira responsablemente.

\section{E:: el caso contratio, curindo la di.} toria no es por inercia sino a excess de In contracion cuando esta d estratos de hiperqunesia ${ }^{-} \mathrm{c}$ huperdina mie uterina de hipertonias generaliza. das o localizadas en forma de anillos de constricción a nivel det cuello o ae un segmento del cuerpo; debe em. pleáse como primera medida la mo: dicación espasmolitica, cuyos reprẹsentantés más importantes los encontrcmos en la papaverina y sus compuestos: el demerol, el betaglicerofosfats sódico-y el sullfato de magnesia, q'e obran disminuyendo los espasmos y la excitación exagerada de la fibra uterina bajando-el tono basal, cuando éste se encuentra elevado. En michas ocasiones el tratamiento es efec. tivo y el parto continúa normalmento: pero en otras la distocia persiste y nos vemos abocados a intervenir en otra forma, especialmente cuando se tratz de enillos de constricción en el cuerpo. segmento o cuello, que no ceden a los espasmolíticos, ni au a la anestesia profunda. Es muy frecuente, por ejemplo, el caso de un cuello espasmódico o que ha llegado a un grado de esclerosks, como sucede a menudo en las primigestantes añosas, en que la dilatación es tán difícll, que el parto se hace-imposible; es entonces cuando se punde emplear con buen éxito en alqunos casos, ta !lamada anostesia en ellla de montar con la que se consiguen a veces efectos sorprendentos, on que un cuello que parece impos!. 
ble de dilatar, se deja hacerlo con rela tiva facilidad bajo esta anestesia; pero con frecuencia vemos también, que este recurso terapéutico fracasa y no hay que insistir ante lo imposible, ye que la dilatación de un evello por $d x$ fuerza puede traet graves conse cumeins, como los descantos amplios o un shock obstetrico qito punde mo ducir la muerte de la madre

Tedo lo anterior, se puede electuot -en un plazo más o ménos cmplio. cuando aún no está en peligro la vida del feto, y la distocia es debida a la dinámica uiterina, es decir, cuando 3 - trata de una distocia materna. Mas cuando unida a la dificultad para el encajamiento, aparece el sufrimients fetal y con él el sufrimiento del obste tra, ya no basta la terapéutica medicamentosa, sino que se impone la term: nación del parto en forma rápida y se. gura. Es cuando entran en juego los tratamientos quirúrgico-obstétricos, lo mismo que cuando el encajamiento no se electúa por brevedad del cordón, ya ésta sea-äbsolutamerne por cordón muy corto o-relativa por presentars ? uria o más circulares, como cuand? hay trastorno del fisiologismo de lo cabeza -y gracias a la cual ésta se $v$ desviada del eje del canal pelvigen: tal.-se orientá mal no se fija bie:i. dando como resultado presentaciones de diámetros-más grandes que dificul. tan el encoriamiento, como - es el eas? de las presentaciones en variedados posteriores, o cuando los movimientos de asinclitismo se hacen más amplic: de lo. normal.

En presencia de una dialocia de en. cajamiento $y$ peligro de una de las -complicaciones que pueden presenta. se, ¿qué debe hacer el obstetra? La res. puesta es lógica: terminar el parto lo mas rapiamente posible $y$ en la tor ma más favorable. poniendo en uego su ciencia y su arte.

Hay varios caminos que conducen en esta clase de distocia a la termina. ción artificial del parto. Son ellos Ia versión interna, la cesárea segmentoria $y$ la aplicación alta de forcens. To dos punden dar buonos o malos resu! todos seaún los casos, segun el crite. rió del medico o según la indicación.

La versión interna tan usada en otros tiempos ha perdido mucho terre. no; -y aun cuando no está completamente abolida, cada día sus partidarios son menores. Ella necesita condiciones especiales como son: que sé trata especialmente de multigestante; que haya suficiente cantidad de liqui. do amniótico; que el útero esté completamente relajado $y$ que la dilatación del cuello la permita, porque de lo contrario nos exponemos a producir una ruptura grave del úfero o a que conseguida la versión, con un cuel!o en dilatación incompleta, al hacer !a êxtracción podálica la cabeza se que de relérida produciéndose la muerto fetat. Con todo hay ocasiones en que ester maniobra da buenos resultados $v$ no rod mos desecharlo por completo. salvo en el caso en que so trate do unz distocier do encajamionto con hi - pertonía uterina muy acentuada, o con gran distensión del segmento, $\mathrm{ca}$ sos eñ tos cuales está formalmento contraindicada, por el grave peligro do producir una ruptura del útero que se. ría algo más grave que la propia distocia que se trata de corregir.

En cuanto a la cesárea segmentaria. no nos cabo duda de que esta es una intervención bion reglada, que da un alio porcentaje de éxitos y que tiene indicaciones sumamente amplias. Mas 
no por ello estamos autorizados para efectuarla y tenerla como la regla en todo caso en que se presente una distocia de encajamiento de causa secundaria. Sobre esta intervención se ha hablado y escrito mucho y se seguiráescribiendo, en razón de sus éxitos. Pero no debemos olvidar que ella constituye un aclo quirúrgico y que como tal, expone a la paciente a los riesgos de toda operación, a más $d .3$ que se trata de una intervención-que se electúa en una mujer que segura mente se va-a ver sometida a emba. razos y partos ulteriores, con un úteró que ya surrió una yez la apertura quirúrgica, dejando como consecuencia una cicatriz que no siempre da segur:dad absoluta de una perfecta consolidación. El médico nunca puede estar seguro de que el sitio de la herida uterina no se vaya a infectar y a producir un proceso de cicatrización defec tuosa y como consecuencia el peligro de una ruptura espontánea en el curso de un embarazo posterior o durante e! trabajo del parto. Tampoco se puedo saber si en un embarazo siguiente se piesente nuevamente distocia - de en cajamiento y como resultado una nue. va intervención cesárea, dejando por tanto a la paciente sujeta a la contin gencia de sufrir una cesárea para cada embarazo. Por otra parte, es posible que una paciente previamente operada de cesárea en sus posteriores partos sea atendida por uñ médico poco experto en la materia, o por un prole sicnal miedoso que crea neçesaria la operación en todo émbarazo por con siderar que si el anterior-fue intervanido, sequramento lo sea en el caso presente, por tratarse de una distocta absoluta o por temor a uná ruptura uterina en el curso del trabajo, sin te- ner en cuenta las consecuencias paud ulteriores casos. En consecuencia, el obstetra debe esforzarse por conseguir la terminación del parto por la vía baja, y sólo cuando ello no sea posible y se vea en la necesidad de ferminarlo por presentarse peligro para la vida de la madre $o^{-}$del hijo, debe efectuar la operación.

Nos queda por analizar el tercer mdio, o sea el tórceps y su aplicación alta; o en cabeza que aun cuando se encuentre fija no se haya encajgdo, es decir, el lórceps alio.

-El fórceps, valioso cónixiliar del especialista que tántas vidas ha aryudado a salvar, no encuentra - reparos en cuamlo se refiere a su aplicación en cabeza encajada y todos están de acuerdo en que es útil y a veces indispensable. Pero cuando se trata de su aplicación alta, surgen las divergencias y en ellas encontramos desde las que lo proscriben rotundamente como un elemento de tortura y sacrificio fetal, hasto los que to defienden y proclaman como el medio más efícaz, cómodo e inocuo. Entre los unos -y los otros están los que lo aceptan con ciertas reservas, aplicándolo algunas v ces cuando lo consideran útil o sustituyéndolo por la cesárea cuando lo imaginan peligroso. Es un hecho qua el fórceps no tue creado para aplicación alta, pero la técnica de los especialistas lo han llevado a tnvadir iz giones vedadas; con éxito en la mayoría de los casos:-

Séa o no creado el fórceps para aplicación sólo sobre cabezá encaiada, es un hecho que sobre el estrecho superior se puede aplicar $y$ - que cuando ello se hace por un especialista de cr!terio, de honradez y de pericia probr. dos, produce éxito. 
Es claro que en todo caso de disto cia de encájamiento, el fórceps alto no es la solución pero en condiciones fa: vorables y llenando los requisitos que tan delicada intervención requiere, se puede inténtar sú aplicación casi cont In sequridad de que en buénas ma nos con ouldado $y$ con las condiciones requericas, se consiguen bumos te sultados especialmente en multios tantes que presenten distocia de enca jamento de causa secundaria.

Aunquie la aplicación - del fórceps pör éncima del estrecho superior es cuestión tan rebatida y en su contra s encuentran eminentes obstetras. quicnes aducen razones de bastante fuerza y criterio, nosotros creemos que la limitación no es tan absoluta y en el!o coincidimos con algunos especia listas, quitenes admiten la posibilidad del empleo del fórceps alto, aduciendo razones también de peso y tan convincentes como lo es el argumento de ha berlo aplicado en diferentes ocasiones con resultados satisfactorios: Es claro que unos y otros tienen razón en sus puntos de vista, pero creemos que así como no se debe proscribir rotunda mente el empleo del fórcéps alto, tam. poco se debe abusar de su aplicación. ya que no todos los casos son tavo. rables a su empleo. Repetimos que nos referimós al empleo del fórceps en distocias de encajamiento de cáusa secundaria, en las cualés tenemos como elemento funciaméntai ia ausen. cla de desproporciones matemoteta. les, o presentaciones trancameñte dis tócicas; partimos de la base de pacien. tes con pelvis normales, con letos tam. bién de tamáno y presentación normáles. En estos carsos bien vale la pe na, en condictones tavorables, hacer ol Inteñto de una aplicación lata, reco. menāando eso si. la suma prudencia que sólo un especialista puede tener. Si el intento fracasa $y$ hay que termi:nar el párlo, que da el recurso de la cesárea. pero si en el intento se sale avante por ese mismo hëcho se ha ahorrado a la paciente una interven cion do mayor categoria $y$ se le ha de jado en condiciones normales para pos. teriores embarazos y partos. Asi lo da muestran las historias que presentamos en el presente trabajo entre-las cúales-hay un buen número de aplicaciones altas de fórceps, con-resultados halagadores si se quiere. Eñ ellas se encuientran también alganos casos de muerte fetal, en los qu no está muy clara la causa de In muerte. Los opositores al fórceps alto podrian decir: la causa no fue otra que la misma aplicación del fórceps; pero éste no es un argumento concluyente. ¿acaso no se podría decir lo mísmo de la cesárea. reconocida por todos como inocua? En muchas ocasiones se hnterviéne pot cesárea con feto vivo y se extrae muerto o en tan majas condl. ciones que muere poca déspués; ¿por qué? Porque se interviene tarde. ¿No podría ser ese el mismo argumento para quitarle al fórceps-alto su apelativo de basiotribo en teto vivo?-El temor a la aplicación alta de fórceps, asi como la esperanza de que logre el encajamiento espontáneaménte, hacen que una gran mayoría de estas intervenciones se realicen tardíamente. cuándo $8 \ominus$ ve lá necesidád de terml. nar el parto en momentós y frente a un gran sufrimiento fetal. El feto noce muerto 0 en muy-malas condictones y entonces se dice: - consecuencia dal lórceps que no se debe apllcar alto. pero no se miden las condiciones en que su aplicación tuve lugar. ¿No se 
produce la muerfe fetal en el curso do un parto espontáneo, cuando se presentá sufrimiento del feto? Con much frecuencia esto sucede y sin émbarao el fórceps no ha sido empleado. Asimismo muchas veces se hace una apt!cación de fórceps son cabeza baja en el estrecho medic o interior y aun en desprendimiento, y sin que of forcens a esta altura sea traumatizants se ol tiene un feto muerto sin que por ello se le pueda achacer la muerte a la aplicación del torceps sino a to tardio de la intervención. Por otrá parte, en cuántas ocasiones un feto-que-sufre fuertemente se salva gracias al fórceps.

No podemos negar, ni queremos hacerlo, que hay casos desgraciados en que una aplicación alta de fórceps ha. ya causado Ia muerte tetal, pero esto no es siempre lo común y hasta pc. driamos asegurar que cuando ello ha sucedido, es porque el fórceps ha sido mal aplicado y su indicación no ha sidó estudiada detenidamente.

Ahora bien, es cierto que el forceps no se puede aplicar en todos los casoe y que para hacerlo, hay que llenar de quisitos sin los cuales es imposible 11 gar a un resultado favorable, y antes por el contrario, producen un tracaso sequro.

En-primer lugar el cuello dél útero. debe estar completamenté dilatado, a que sea posible completar su dilata. ción manualmente, porque de lo contrario el fórceps no se podría introdu. cir y en caso de hacerlo, sería a costa de desgarros, éstos si amplios y gra ves con peligro igualmente de désgaro del segmento.

En segundo lugar, hay que tener seguridad absoluta de que no existe desproporción celalopélvicá, ni siquio- ra minima o dudosa; antes bien estar plenamente convencidos de que la cabeza-cabe-sin quedar apretada en el estrecho superior, para que ain con el aumento de diámetro próducido por las cucharas de forceps, el paso sea posible facilmente. De otra manera se iracasa-y entonces si hay pelute para

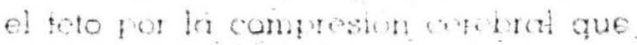

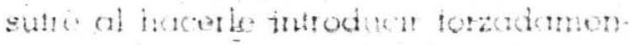
to la catrza en la pelyas.

En tercer lugar, las tracciones ejercidas-sobre el tórceps-no han de ser violentas, con êl-fin dê-hacer encajar y-descender la présentación a la viva fuerza y por sobre-todo, sino-que deben ser tracciones suaves, muy prudentes, dirigidas hacia abajo y hacia atrás, buscándo el eje del encajamiento $y$ en el momento de la contraceión si la hay para lo cual puede ser útil hacer suspender la anestesia, y-teniendo el suficiente carácter para desistir de la extracción del feto por abajo, si el encajamiento ofrece alguna dificul. tad. Por ello debe intentarlo el verdadero especialista que lógicamente searrepentirá anté el fracaso en contra del médico-genèral, quo tendrá más probabilidad de no hacerlo $y$ entonces se obsesionará con el torceps y no ve más solución que ésta.

En cuarto lugar; hay que tener la precaución de hacer la aplicación del fórceps en un medio quirúrgico, teniendo listo todo lo necesario para cambiar rapidamente ja iniervencion- pōr una cesárea, si al hacer el intento de-exfracción con el tórceps, se nota dificultad, aun cuando sea minima, para conseguirló, teñiendo en cuenta la obligación de salvaguardiaf la vida del feto y la integridad de la madre, por to-cual consideramos un-errór tratar de sacár un teto a loda-costa pór el 
sole thro a cren qu und $v a$ int

ducido el forceps, no se debe sacar vacio. Repetimos que la aplicación al* ta del fórceps es intervención muy de. licada que tiene que hacerla un especialista avanzado en la materia y quẹ sólo debe ser uñ intento en que si re. sulta con facilidac el tiempo del enca. jamionte. se puedo continuar la ope racion, pero que si hay dilicultad por leve que ella sea, se debe tambiar la intervención por versión o por cesárea. Por eso no lo aconsejamos para el medio rúal.

- En quinto lugar, el operador debe disponer de un ayudante práctico para qué éste le facilite las maniobras que debe efectuar al hacer la toma y las tracciones.

En sexto lugar debe tratarse de hacer una toma lo mejor posible, para evitar el sufrimiento y la compresión de la cabeza lo más que se pueda. Para ello, como la presentación por lo general antes del encajamiento, se orienta en el diámetro transverso de la pelvis y como en esta posición no se puede hacer una toma directa potque resultaria fronto-occipital, se puede hacei una toma oblicua, -frónto-mastoldea que es buena aplicando el fórceps según las técnicas clásicas, como para una variedad de posición antertor: pero todavia es mejor emplear una pequeña maniobra que da muy buen resultado y que consiste en que el ciruiano, antes de introducir la primera cuchara y con la misma mano que le va a-servir de guia, rote la presentación a O. P. a O. S., mientras que el ayudante la flia sólidamente en esta posición por la pared abdominal para mantenerla fija e impedir que se vuel. va a O. T., en estas condiciones y 8 f el ayudante cumple blep su función,
So pued nacer una toma difecto ideal. Entonces una-vez hecho la to. ma con punto de apoyo en el tórcops se rota la presentación a una vartedad de posición oblicua $y$ en éstas condiciones sc empiezan las tracciones pa ra el encaiamiento. Incluso se pueden hacer hasta dos $y$ tres intentos de aplicacion. suavemente destinades a corregir las tomas defectuósas que ha. yan resultado a impedido el encaja. miento perfecto.

En séptimo lugar hay necesidad imprescindible de hacer una revistón manual del útero, cuello $y$-vaginas, una vez terminada ta-intervención, para cerciorarse que no han quedado lesiones maternas, y si hay desgarros suturarlos inmediatamente, ya que son causa frecuente de hemorragia y shock.

Sobra advertir que todas estas -maniobras tienen que hacerse bajo cmestesia profunda, porque son dolorosas en extremo y sin anestesia se corre el peligro de shock.

Es bajo estas condiciones que con. sideramos posible la aplicación, o me jor el intento del lórceps alto, para terminar rápidamente un parto y muy especialmente, euando se trata de fotos pequeños en mujeres de pelvis muy amplias que permiten tácilmente el encajamiento y los movimientos del lórceps. De lo contrario el tórceps alto no debe intentarse, nl tom slquiesa pensarse en ello, porque-entences á demaslado peligroso. Creemos que los casos de exito que presentamos y los que relatan quienes aceptan el tárceps por sobre el estrecho superior, se do ben a que se han seguido estos-requisitos y se han conseguldo las condiclones favorables para hacerlo. 
Estado actual del fórceps sobre el estrecho superior y su relación con la distocia de encajamiento de causa secundaria.

No queremos terminar el presente trabajo: sin hacer antes un análisis sobre el forceps y sobre los argumen. los que se aducen en pro y en contra de sú aplicación por encima del estre. cho superior.

El lórceps como su nombre ló indica. es una pinza que cumple una tinalidad doble presión y extracción y está destinado para aplicarlo sobre la cabeza del feto para extraerlo de los genitales. Desde su invención por Pedro Chamberlen en 1600 , ha venido prestando grandes servicios a quienes se-dedican al ejercicio de la obstetricia.

En un principio su empleo quedó limitado a la familia del inventor, que guardó el secreto por mucho trempo: más tarde, conpcido sú uso y discutida:su utilidad, se hizo común al protesional, hasta convertirse casi en un -símbolo del partero. Désde entonces el primitivo aparato ha sufrido modificaciones en sur estructura pero no en su finalidad. Constituido por dos ramas, que a la manera de dós manos cogen la cábeza sólidamente para ex traerla, fuerón ideadas modificaciones a su estructura, hasta conseguir diferentes tipos de fórceps que conservando las curyaturas primitivas de sus clicharas difieren en cuanto a la disposi. ción de sus-ramas; teniéndose así los fórceps de ramas cruzadas y los de ramas convêrgentes. Los más usados entre-los de ramas cruzadas son los modelos de Tarnier, Simpson y Kje. lland $y$ entre los de ramas paralelas el de Demelin.
Entre nosotros los mus empleado. son los modetos de Tarnier y Simpson. en especial este último, del qư existen varios tamaños pará su aplicación, segün la altura a que se encuentre la cabeza fetal.

No cabe duda de que la introducción del forceps a la práctica obstetrica represento uno de los mayorés pro gresos en esta rama de la medicina: pero tampoco es menos cierto que on un principio se empleo con abuso, abuso que ha ido corrigiéndose pau- latinamenter y que hoy día gracias a la experiencia que de él se tiene y de los estudios llevados a cabo, su empleo tiene normas - y leyes que hacen del fórceps un instrumento casi inocuo, necesario y a veces indispensable, Hegando a tener indicaciones precisas que ha hecho que se impongá en la práctica obstétrica moderna como un instrumento vèrdáderamente insustituíble.

Como los argumentos son controvertibles, haremos un parálelo-entre las razones en pró y en contra de las aph. cáciones altas de-fórcèps.

Primer argumento. El bacinete: se asegura que el défecto o talta de en cajamiento equivale a pelviviciación: si ésta existe, absurdo será aplicar fórceps y el fracaso será total, pues si la cabeza no pasồ sola, mucho me. nós lo-hará aumenfando su- esfera u ovoide con las cucharas del aparato; esto sin contar ötras consecuencias: el fraumatismo fetal, el irremediable des. Hzamiento de presión o traceión.... El argumento es poderoso e irrefutable, hoy en día no ofrece duda y ante una pelviviciación franca sin enca. jamiento, con prueba de frabajo ca. bal, la conducta la ríge la cesćrea seg. mentaria. Pero este no es el campo de 
la distocia de encajamiento que nos ocupa, el grupo que hemos analizado descarta la pelviviciación, la respeta. no invade su fuero; mas si no existe pelviviciación y la distocia do enca jamiento se debe a alguná de las cau. sas estudiadas, y a ello se ha llegado después de un estricto diagnóstico cli nico cbstétrico, y existe la necesidad de terminar el parto artificialmente, la aplicación de fórceps en el estrecho superior 0 arriba de éste se debe in. tentar pero con gran conocimiento de cousa respecto a la acción y resultan. te: con la más rigurósa prudencia, inclusive ordenando simultáneamente la preparación para probable cesárea, con un cuidadoso juicio y raciocinio de diagnóstico y conducta que permita el intento con una recíproca racional: saber curepentires a tlempo, no insistir ante lo imposible, tomer al trau. matismo obstétrico, no hacer una lo cura obstétrica, no convertirse de partoro prudente y lleiólófico on un pal. cópata del parto. obsesionándose con una sola idea fija, a saber: se inter. vino por vía baja, luego el parto-ten. drá que terminar por ésta, pase lo que pase. gástese el tlempo quie se gaste. hágase el traumatismo que se haga. el feto no vale en su vida, lo que vale es sacarlo al mundo.

Segunde argumento. El encajamiento condición para aplicar fórceps. Es de la hermenéutica obstétrića fijar como condición necesaria para aplicar for ceps, que la presentación esté encajada con la circunferencia mayor de lá cabeza fetal abajo del estrecho supe -rior para que asi la manipulactón del aparato y sus movimientos iniciales $y$ siguientes evolucioñes se verifiquen libremente en el amplio espacio de la excaración pélvica, en donde se ob- tendrá éxito: todo lo contrario de cuan. do se actua en el estrecho superior que por detinición traduce un espacto reducido que anatómicamente da entrada a la excavación o región ensanchada del bacinete.

El argumento tiene solidez; pero relativa, pues vale si se relaciona con el argumento primero de la pelvivicia ción: pero en un bacinete de dimensio. nes normales con el tipo de distocias de causa secundaria no tiene valor absoluto, ya que no hay problema para aplicar fórceps porque el estado del polo cefálico ante el estrecho superior es el mismo o semejonte al que prosentaria frente al parto espontáneo quie se desarrolla de acuerdo con la euto. cia, y por otra parte, no hay impedi. mento físico para colocar las cucharas del fórceps en el área del estrecho superior cuando-el bacinete es normal. Además, la experiencia ha ratificado dos hechos: que mucho se yerra en la apreciación del encajamiento do su grado, de su profundidad, y que slempre se ha seguido aplicando forceps - y se ha logrado haces pasar el volu. men-cabeza-cucharas.

En cuanto al primer hecho ha sido común diagnosticar una cabeza como encajada y después llegar a la conclusión de que aún es movible o re chazable, error que se debe a que se hace el examen en el momento de una contracción uterina, a la bolsa serosan. guinea o a talta do práctica y que se aclara cuando en el momento de intervenir-por vía vaginal, se aneistesia a la paciente; hay o no relajación ute: rina, pero lo clerto es que la presentación asciende porque se hace recha. zable y movible tácilmente.

Hoy está demostrado que dobe pecar por exceso y no por dofocto on 
cuanto al diagnóstico del encajamien to. pues todas las presentaciones insinuadas o fijas, son cesareables y no están perfectamente encajadas; sólo existe encajamiento verdadero, cuando el límite inferior de lá cabeza, prescindiendo de la bolsa serosanguinea llega al tercer plano de Hodge y está muy próxima a este la gran circunfe. rencia cefálica. Los casos intermedius a los paralelos primero y tercero, no traducen sino escalas intermedias do encajamiento, y són todavia cesarea. bles con dificultad ligera mediana o grande, pero siempre cesareables.

En cuanto al segundo hecho y en correlación con el primero, a pesar del error en el encajamiento. cuando se ha resuelto terminar el parto con fórceps, este se ha logrado, al fin $y$ al cabo, por lo menos en la gran mayoría de las veces en un período corto o largo, con aturdimiento o- sin éste, pero se ha logrado sin grave morbilidad materna.

Tercer arqumento. La toma obstetri. ca. La técnica más inexorable éxige que al aplicar las cucharas del tórceps se realice la buena presa, con toma ideal, biparietal o biauricular, con las cucharas en regiones simétricas dol cráneo y de la pelvis y con el eje del aparato en dirección del diámetro occipito-mentonero. Lograr lo anterior será lánto más difícil cuanto más baja esté la cabeza y sérá un imposible en el estrecho superior o arriba de este, porque se supone la cabeza en variedad de posición transversa, que es lo más trecuente y las tres tomas conocidas en drán el siguiente resultado: la biparie tal no se alcanzará y si por casuatidad se obtuviere, el encajamiento $y$ extracción se obstaculizarian porque se haric un puente promonto-coxígeno con la rama posterior, se eliminaría la curvatura sacra tan necesaria en el mecanismo fisiológico del parto y entonces no se podría imitar éste; la oblicua fronto-mastoides podrá realizarse $\alpha$ costa de vencer multiples dificultadés. debido a que serían reducidísimas para alojar las cucharas, las hendiduras de los extremos de los diámetros obli. cuos, y además en el foto productrian morbilidad por las paralisis taciales debidas a la comprensión del agujero estilo-mastoideo; la antero-posterior, fronto-occipital, tendría las mismas dificultades con mayor intensidad y pro - vocaria gran reducción del diámetro occipito-frontal con consiguiente au. mento- del biparietal que no podrá franquear el diámetro-útil y lo cual hará además lesiones encéfalo-craneanas de pronóstico fetal grave, inmediato o futuro. Finálmente, por el asinclitismo-del polo cefálico; nunca-se hará una satisfactoria colocación de las cucharas.

- Los argumentos anteriores se refutañ diciendo que en el tipo de disto ela de encajamiento que nos ocupa. se deben descartar las tomas antero posteriores por su agresividad. La to - ma ideal es de suma dificultad conse guirla ya que si no se alcanza estan do la cabezá cerca del estrecho infe rior muchas veces aun cuando se haga la-más completa hilación de pro yecto de-iórceps, con menor razón sérá ícil estando la cabeza a la aitura del estrecho superior. No obstante algu. nas veces se logra una toma deal, cuando es posible emplear un peque ño truco que consiste en rotar previa mente la cabeza a O.P. o a O. S. con la mano que ha de guiar la primera cuchara y una vez obienido esto, un ayudante mantiene la cabeza en esta - posictón, cogiéndola solidamonte con 
ambas manos por la pared abdom:nal; sin sacar la mano guía, mientras que ésta ayuda a mantener la cabe. za én la posición que se le ha dadas. se introduce la primera, directamente, para una aplicación directa y una vez colocada se-introduce la segundr rama lográndose muchas veces ha cer una toma ideal como lo hemos visto. Una vez lograda lá toma, se rota ligeramente la presentación con el lórceps a una variedad de posición oblicua y se - inician las - tracciones para el encajamiento. . Ahora bien, si no se logra una toma ideal la toma oblicua que resultare no tiene inconveniente porque si la pelvis materna es de amplitud normal, no habrá dificultades para dar eavida a las cu= charas, y además las- lesiones fetales que ocasiona dicha toma se restituyen ad integrum y tienen la mismi suerte de las que se presentan en las mismas tomas realizadas abajo del estrecho superior, tomas que por clerto son muy frecuentes por muy desSendida que esté la presentación.

En el argumento que se comenta. falta abrir también le discusión sobro los artificios y sobre los modelos do lórceps destinados a torregir las difi cultades para hacer la toma ideal en el estrecho superior o arriba de éste y solventar los inconvenientes mecá nicos y físicos de las curvas de los aparatos, para-relacionarse côn lis conliguráción del bacinele; a) Maniobre de Jarnes Massini bf Fórceps de Kjelland; e) Fórceps de Demelin S.

a) Maniobra de james - Massini. Este distinguido Profosor argentino, para lograr la foma en que el eje dol afocarato siga el eje occipito mentone. ro tetal, y partiendo de la base de que la cabeza arriba del estrecho superior está en una variedad occipt. to-transversa, -izquierda ó derecha de flejada, recurre a artificios ingentosos y hace la toma invertida, ideal y flo xora, que lleva su nombre al tiempo que-da a la mano guia un papel opuestc al que tiene en la aplicarion clásica y además asimila ta varte dad transversa a la correspondiente posterior para las siguientes manio bras. Es invertida porque la curva pélvica del aparato mira no al oce pital sino hacia la frente, tanto en el momento de hacer la aplicación coms una vez ferminada ésta. Es ideal por que coinciden el eje del aparato y al occipito mentonero. Es flexora porque mediante un movimiento de pa lanca, cambia la actitud de la cabe. za y se provoca-la flexión. La mano guía es única y lleva el nombre contrario a la variedad de posición.

Ejemplo: sea una O. I. D. T. deflo jada sobre el estrecho superior. $\mathrm{H} \rightarrow$ chó el proyecto de fórceps se procedo así: introducción de la rama anterior - dérecha conducida por la mano dorecha y guiada con la izquierda: s-a lleva por la articulación sacrollíaca derecha y se termina su colocación con la maniobra do La Chapelle, he. cha por delante del occipital y con la cual se adapta a éste la curva celáli. ca. El mango del tórceps-quedará contra el muslo materno derecho. In. troducción de la rama posterior o iz quierda directamente llevada por la mano dérecha y guiada por la misma mano guía, o sea la izquferda; con lo cual se adapta la curva cefálica al parietal posterior- Articutación del fór. ceps, previo descruzamiento, después de to cual los mangos de éste queda. rán contra el muslo derecho de la mo. dre. Elexión de la cabeza para to 
cual bastarú llevar los mangos del aparato contra el muslo izquierdo de la madre. Asimilación a-O. I. D. P. para lo cual se hace la primera tracción, se lleva el occipital al seno sa cro-iliaco derecho y ya quedara la presentación orientada en el diame tro del encajamiento de la O. I. D. P. La futura conducta será la de esta pre sentación encajada y así seguirá descenso, rotación a O. P. seguida di. desarticulación del aparato para ha cer-nueva aplicación en el estrecho inferior para la última variedad de desprendimiento y de acuerdo con las normas clásicas.

b) El Fórcops de Bjelland. Sư prin cipio fundamental es hacer la toma ideal mediante la maniobra de la rotación in situ de la rama anterior.

Algunos lo han aplicado en la va riedad de posición transversa sobre el estrecho superior, teniendo en cuenta la siguiente técnica: la aplicación se hará en el sentido anteroposterior del bacinete. Siempre se tomarán las ramas-del fórceps con la mano de recha y a modo de bayoneta. Se in troducirá primero la rama anterior con la curva cafálica mirando hacia ul pubis, cuidadosamente guiada por lr: mano izquierda que será una sola y misma mano guía. Rotación in situ. rotación intrauterina de-la. cucharc del fórceps, -para que la curva cefálica se adapte al parietal anterior, que ya en el tiempo anterior estaba adap tada al pubis; dicha rotación se he ce a través del mango, con mucho survidad y on el sentido de la curvo pélvica del forceps o de un botonelto especial que tiene. Aplicación de Ir rama posterior, en sentido anteropos. terior, llevando la cuchara - contra el promontorio hasta adaptar su curva cefalica al parietal posterior y termnar con la articulación a la rama anterior. Podrá quedar una cuchara más profunda que la otra (asinclitismo) y esto lo permite la amplia corredera d. articulación. Tracciones de encaja miento y descenso. Rotación de 90 grados espontánea o provocada $y$ desprendimiento en O. P. o nueva apli cacion para O. P., previa desarticula ción del aparafo.

c) El Fórcops do Domolin 8. Tan co nocido y tan bien estudiado en la te sis del doctor Aristóbulo Urrego, tam bién se le ha reconocido gran utilidad en la operación alta de fórceps, especialmente cuando se introduce primero la rama anterior, porque es un fórceps que imita el mecanismo del parto espontáneo, facilita la liber tad de movimientos de la cabeza, pro voca la flexión, corrige el asinclitismo o éste no dificulta su aplicación y articulación, hace una buena presa, la toma es sólida por ser sus ramas con vergentes, porque sus tracciones se hacen en la forma más científica, etc.

\section{Críticas}

a) El James Massini merece enco. mio por su ingeniosidad y en teoría representa una innovación o renover ción. Pero en la práctica posiblemen te no da los resultados que se propo ne, pues es difícil que se tenga éxilo en-cada uno de sus tiempos, lan con. irarios a los que ya han productio lamiliarización al tocólogo. En el maniquí de Obstétrica es un lujo do intervención y todos sus tiempos que constituyen una serie do artificios se ierlizan à la perfección.

b) El-Kjelland no se ho indicado sino especialmente para las, variedodes de posiciones transversas, encaja. 
y si su campo de-acción ha subido hasta el estrecho superior o arriba d éste. no ha sido tal su objeto princi. pal, sino et déseo de ensayarlo en lí operación alla de fórceps.

c) El Demelin 8. Sirve mucho más èn to oneracion altio de forceps cumd In cabora esta a la alfura del estr cho sumerior: esto hay que recono cerlo. Lastima que sea un apara! tan grande de cucharas tan emplias en caso contrario, daria mejor rendi miento:-

Ante la mániobrá de Jamés Masini. ante- los fórceps de Kielland y Deme. lin 8, se debe preferir el fórceps de Simpson modelo pequeño que se comporta como un gran fórceps en la operación alta, en donde merece la simpatía y te que se le tiene en las regiones más declives del bacinete.

Cuarto argumento. Se ha dadoveto al fórceps sobre el estrecho superior porque a esta altura pertenece el radio de -acción de la cesárea y de la versión podálica; el modernismo oblétrico por la inocuidad de la primera, asegura que el balance materno fetal favore cé a la cesárea y perjudica al fórceps alto. Además los autores antiguos pre lerian la versión al fórceps aunque en ella el resultado fetal llegare hasta ta mortalidad, pero en todo caso la con. sideraban mejor para ta madre. Hoý lódos verieramos a lá cesárea seg. mentaria $y$ reconocemos sús infinitos beneficios, pero en algunas cistocias dé encajamlento,-el fórceps puede sus tituirla con ventaja, sustitución que es de-rigor o de emergencla salvadora cucindo la vitalídad del teto se com-promete lánto, qué no da tiempo para la preparación y etecución de una cesárea (en lo cual pascría por lo mé. dable practicat on feto-muats en po encia o-en acto o on lapes humedia to $y$ a la cual no es justo nt cientifico exponet a la madré para tener un hilo que no-ha de vivir.

Quinto arqumento. El fórceps allo esta reservado a los prácticos y es pecialistas en obstetricia clinica. Este si es un rgumento-convincente, por que la aplicacion exige conocimien to de causa y habilidad en la postu ra de las cucharas que sólo la da ta experiencia, y por sobre todo esto: es más probable que el especialista sepa arrepentirse-a tiempo -y que tenga el suficiente criterio para-cambiar la conducta por una cesárea si llega el momento en que sus intenciones de aplicación no han tenido éx:to y que na le tema al qué dirán. y no se amilane ante la supuesta crifica por cambiar una conducta en que hubo fracaso.

A lo que no se debe-liegar-es al extremo apasionado de algunos au. tores que al declarar que el fórceps es una operación contra natural que de be ser patrimorito del obstetra áveza. do de la cual debe abstenerse el poco práctico, el iobstetra rural., acon seian a estos últimos la embriotomía on toto vivo: craneolomia y basiotrip: sid, cuyos nombres encrespan y horripilan, aunque ellos aduzcan en forma tan discutible y etrada to slquiente: -el fórceps alto que logra extraer al feto no es sino una embriotomía disi. mulada y vergonzante con todos sus inconvententes y ninguna de sus veñtaicis.

\section{Conclusiones finales}

El parto es un acto fistológlco,-regl do por leyes biológicas que constitu yen la fisiología obstétrica. 
Llegada la preñez a su término, so instala el trabajo de parto, el cual si ps normal, da origen al parto eutócico o fisiológico.

Cuando el fisiologismo del parto se ve alterado en todas o en una de sus partes, aparece la entidad denomine da distocia.

Existe una distocia primitiva $0 a b$ soluta en la que el parto es imposib!. por tas vías genitales, y una distocin relativa del encajamiento, dependien te de diferentes corusas que sí puedo. permitir la realización del parto por via baja y que constituye la distocia de encajamiento de causa secundaria.

La distocia de encajamiento de causa secundaria puede tratarse mé. dicamente, obteniéndose a veces buenos resultados con los ocitócicos n con los espasmolíticos, según el caso; Cuando el tratamtento médico fracasa, hay que recurrir a otros métodos como la versión, la cesárec, o el fórceps alto, para salvar: la vida de! feto que casi siempre se ve comprometidg.

La aplicación alta del fórceps está contraindicada por unos obstetras $y$ aceptada por otros. Es una intervención que se puede emplear coñ bue nos resultados, siempre que se llenen los requisitos indispensables para poder eimplearla.

El tórceps alto debe emplearse en un medio quirúrgtco, teniendo listo todo lo necesario para cambiar la coriducta por una cèsárea, -st tracaśr of intento.

No -se debe insistir ante lo imposi ble y se debe lener el suficlente carác. ter para desistir de la intervención, se aprecia dificultad.

La aplicación-ella de fórceps, sólo debe intentarla un especialista en la matcria y con larga práctica. Esta inlarvención está contraindicada en la inminencia de ruptura así como la versión y en este caso sólo debe em plearse la cesárea.

En presencia de una distocia de en. cajamiento de causa secundaria hay que hacer un estudio cuidadoso do la cause $y$ proceder de acuerdo con un criterio científico, resolviendo la con ducta obstétrica, antes de que sea de. masiado tarde, pues la vida del feto se encuentra seriamente amenazada.

Nuestra experiencia en historias clínicas demuestra que el fórceps pue de invadir el campo de la cesárea y no ser una embriotomía en feto vivo.

\section{BIBLIOGRAFIA}

Acosta José del Carmen-Conferer cias de Clínica Obstétrica.

Acosta Hernando-Conferenciās de Obstetricia.

Molica Carlos Julio-Conterencias d? Clinica Obstétrica.

Peralta C. Rafal-Conterencias do Obstetricia.

-Peralta C. Rafael- "Colombia Méd:= ca*. Vol. III, 1944 № 8. «A propósito do las aplicaciones de fórceps so bre el estrecho superior.

Botella Llusia José-Patolóaía Obste trica. Primera edición.

Carl-Henry Davis-Gynocology and Obsietrics.

Doderlein A.-Tratado de Obsletricia. Segunda edición.

De Lee Grinhill-Principios y Práctice de Obstetricia. Octava edición.

Pérez Manuel Luis-Tratado de Obste: tricia. Quinta edición.

Recasens Girol-Tratado de Obstefri. cia. Séptimá edición. 